\title{
Modeling of Handover Process in Operation Control System of Maglev Train Using Timed Automata
}

\author{
Gegerile, ${ }^{*}$ Hongze Xu, Yelei Li and Jianfeng Liu \\ School of Electronic and Information Engineering, Beijing Jiaotong University, No.3, Shangyuancun, Haidian district, Beijing, \\ China \\ *Corresponding author
}

\begin{abstract}
The OCS (operation control system) is playing a vital role in ensuring the high efficiency and safety of the maglev train. In this paper, we focused on modeling and verification of the handover process of OCS. After we analyzed the function and performance of the system, the process was modeled as a network of timed automata by the verification tool UPPAAL, which was synchronized to describe the communication establishment and massage exchange among subsystems. Then, some key properties that are vital to guarantee the correctness of the handover process were verified with the UPPAAL. Finally, the result shows that the handover process in operation control system of maglev train is provided with safety and restricted activity.
\end{abstract}

Keywords- timed auotomata; maglev train; operation control system; handover

\section{INTRODUCTION}

As a new type of transportation system, the maglev train has the advantages of energy saving, environmentally friendly, low noise and so on [1]. The OCS (operation control system) of maglev train is playing a vital role in ensuring the high efficiency and safety of the train in such a high speed at $500 \mathrm{~km} / \mathrm{h}$. When the maglev train runs through several districts, the operation control system needs to switch from one DCS (Decentralized Control System) to another frequently [2]. The handover process of DCS is one of the main factors that severely influence the efficiency, reliability and safety of the system. Thus the formal modeling and verification of the handover process of the maglev train operation control system is of great importance.

At present the research on maglev train is focused on the field of levitation system and motor control. While, there is less research on the operation control system, especially the modeling and verification of system. As for handover process, most of the research focused on the modeling of RBC (Radio Block Centre) of CTCS-3(Chinese Train Control System level 3)[3-5]. After the analysis, we build a model of the handover process of maglev train operation control system using timed automata theory in this paper. Then the simulation analysis is carried out by means of timed automata model verification tool UPPAAL.

This paper is organized as follows: in Section II, we analyze the structure and the function of maglev train operation control system and describe the handover process of the system. Timed automata and its verification tool UPPAAL are introduced in Section III. Then we give a description to the modeling and verification of the handover process in Section IV. Finally, conclusions are given in Section VI.

\section{THE OCS OF MAGLEV TRAIN}

The OCS completes the function of safety protection, automatic operation and dispatching management, cooperating with the subsystems and devices like vehicle, traction system, route and switch.

\section{A. The Structure of OCS}

The OCS in Shanghai maglev line and TransRapid in Germany is divided into 3 levels which consist of 4 subsystems (as shown in Figure 1): Central Control System (CCS), Decentralized Control System (DCS), Vehicle control System (VCS) and the communication system.

CCS, which is located in control center, consists of ATO (Automatic Train Operation), OTS (Operation Terminal System) and DTS (Distinction Terminal System). The main function of this subsystem is to work out and execute the train operating plan and to supervise the train running.

VCS is made up of vehicle safety computer (VSC1, VSC2) and vehicle transfer computer (VTC) installed in both ends of the train. This subsystem mainly complete the function of positioning train, monitoring train speed curve, forced stop management, train suspension and so on.

DCS, usually located in the traction substation or switching station, mainly includes decentralized control computer system (DCC), decentralized safety control system (DSC), decentralized power switch system (DPS) and decentralized switch model (DSM). The main function is to complete the

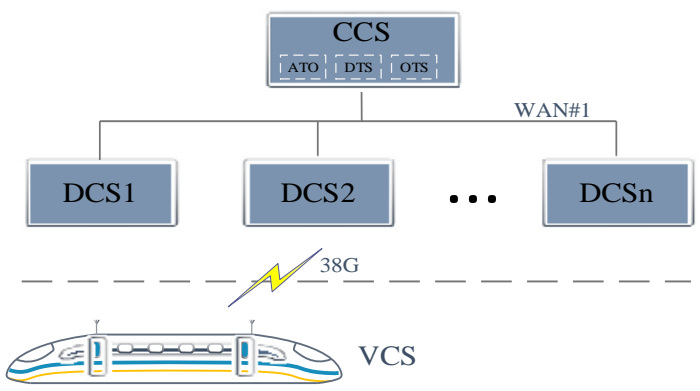

FIGURE I. STRUCTURE OF OPERATION CONTROL SYSTEM 
control and protection of train operation in the district and handover process between the adjacent districts, cooperating with VCS. Communication subsystem is a distributed information transmission and control network platform, which has prosperities of safety, reliable, high speed and redundancy. It consists of WAN, interlocking bus and $38 \mathrm{GHz}$ wireless system.

\section{B. The Handover Process of DCS}

The handover management of DCS is a process that hands over the train from the dominant of one DCS to the dominant of the next one. As shown in Figure 2, when the train runs to the boundary of current DCS, it should complete the handover of the DCS. Except for the safety cut-off function of traction power supply, the control and protection related to handover process can be conducted by a non-interactive way.

When the train runs to the starting point of handover zone, the adjacent DCSs exchange related message (including train parameter, train position, line data and stop point). The DCS which receives the message, should save all the data required for handover temporarily. The instruction of the speed curve monitoring module of the VCS triggers related operation of handing over. The current DCS sends handover instruction to the DCS in the next district. Only when the next district returns a successful handover message, the current one cuts off the control and protection of the train. If there is a connection failure or communication failure during the process, the adjacent districts cut off the traction power supply independently, besides the speed curve monitoring module of the VCS will receive the corresponding message.

The main functions that system should complete during the handover process are stop point stepping and safety responsibility handover. Unlike traditional train, in maglev train system each train has its reachable stop point, the operation control system should ensure the train can reach the current stop point in any case. After reaching the current stop point, the OCS should set the next reachable stop point on the reserved route as the current one, which is called stop point stepping. In the course of handover, current stop point and the next stop point are in different districts. Besides the system should complete the handover of train protection, route

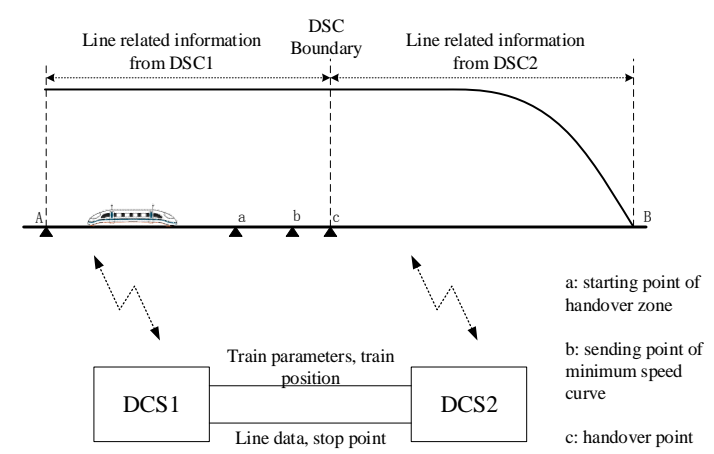

FIGURE II. INFORMATION TRANSFER OF DCS HANDOVER PROCESS protection, speed curve monitoring and traction cut-off.

\section{TIMED AUTOMATA AND UPPAAL}

\section{A. Timed Automata}

Timed automata is a theory for modeling and verification of real time systems [6]. A timed automata is a finite-state machine extended with a finite set of real-valued clocks.

Formally, a timed automaton can be defined as a tuple $A=\left(L, L_{0}, A, C, I, E\right)$ that consists of the following components: $L$ is a set of locations, $L_{0}$ is the initial location, $A$ is a set of actions, $C$ is a set of clocks, $I$ is a set of invariants on the location, and $E \subseteq L \times A \times B(c) \times 2^{c} \times L$, gives a set of transition edges.

$B(c)$ is a clock constrain, ranged over by g later, which is a conjunctive formula of atomic constraints of the form $x \sim n$ or $x$ $-y \sim n$ for $x, y \in C, \sim \in\{\leq,<,=,>, \geq\}$ and $n \in N$. We shall write $l \stackrel{g, a, r}{\longrightarrow} l^{\prime}$ when $\left\langle l, g, a, r, l^{\prime}\right\rangle$. The edge connects two locations with an action, a guard and a set of clocks to be reset.

The semantics of a timed automaton is defined as a transition system where a state or configuration consists of the current location and the current values of clocks [4]. There are two types of transitions between states. The automaton may either delay for some time (a delay transition), or follow an enabled edge (an action transition). The states are pairs $<l, u>$, and transitions are defined by the rules:

$$
\begin{aligned}
& \langle l, u\rangle \stackrel{d}{\longrightarrow}\langle l, u+d\rangle \text { if } u \in I(l) \text { and }(u+d) \in I(l) \text { for } d \in I R_{+} \\
& \langle l, u\rangle \stackrel{a}{\longrightarrow}\left\langle l^{\prime}, d^{\prime}\right\rangle \text { if } l \stackrel{g, a, r}{\longrightarrow} l^{\prime}, u \in g, u^{\prime}=[r \mapsto 0] u, u^{\prime} \in I\left(l^{\prime}\right)
\end{aligned}
$$

\section{B. UPPAAL}

UPPAAL is a tool suite for automatic verification of safety and bounded aliveness properties of timed automata networks [7]. The tool was developed as the result of intense research collaboration between BRICS at Aalborg University and Department of Computing System at Uppsala University. The two main design criteria for UPPAAL has been efficiency and ease of usage.

A system in UPPAAL is composed of concurrent processes, each of them modeled as an automaton. The automaton has a set of locations. Transitions are used to change location. To control when to take a transition, it is possible to have a guard and a synchronization. A guard is a condition on the variables and the clocks saying when the transition is enabled. The synchronization mechanism in UPPAAL is a hand-shaking synchronization: two processes take a transition at the same time, one will have an "a!" and the other an "a?" with a being the synchronization channel. When taking a transition, two actions are possible: assignment of variables or reset of clocks.

UPPAAL consists of three main parts: a system editor, a simulator and a verifier. The system editor is used to create and edit the system to be analyzed. A systems can be described as a 
series of process templates, some global declarations, process assignments and a system definition. The simulator is a validation tool that is used to check if the execution of the system model may be in error, so as to detect some errors before verification. The verifier is used to check the clock constrains and react limiting properties. It also provides a specification editor for system requirements specifications and documents.

\section{MODELING AND VERIFICATION}

\section{A. Modeling of Handover Process Based on Timed Automata}

The main subsystems communicate in the process of handover are VCS and two adjacent DCSs. The timed automata model of the system can be described by the product of the following three modules. TA = VCS $\|$ hov DCS $\|$ accDCS

As shown in Figure 3, 4 and 5, each of the module is defined based on timed automata and using UPPAAL. The synchronization of the modules is accomplished by the channels, besides the model defines many global variables and local ones to ensure the validity of the system logic.

\section{1) Modeling of VCS}

The VCS is responsible for monitoring the train speed curve and controlling the train by communicating with the ground system periodically. During the process of handover, it should establish communication with the acceptance DCS, and exchange massage with both of the DCSs at the same time. Then after the train leave the boundary of handover zone, VCS will disconnect with the handover DCS and keep connecting with the acceptance one.

As shown in Figure 3, we use two Boolean variables (hov_VCS and acc_VCS) to decide whether to connect with the handover DCS and acceptance DCS respectively. VCS send massage to the DCSs via synchronization channels "vcs2Hov!" and "vcs2Acc!" then receive massage from DCSs via "hov2VCS?” and “acc2VCS?”.

\section{2) Modeling of handover DCS}

The handover DCS is responsible for route protection and train management (including information about train location, train state and state of radio connection) by communicating with the VCS and acceptance DCS. When the train reaches the handover zone it starts to exchange related massage with

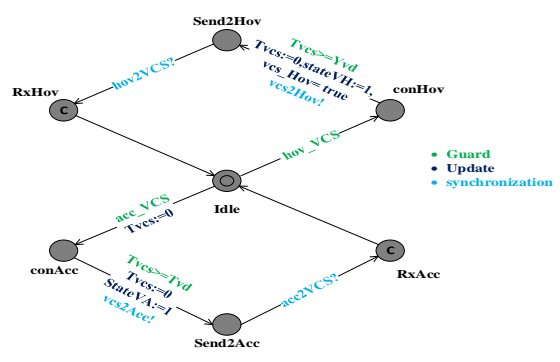

FIGURE III. TIMED AUTOMATA OF VCS

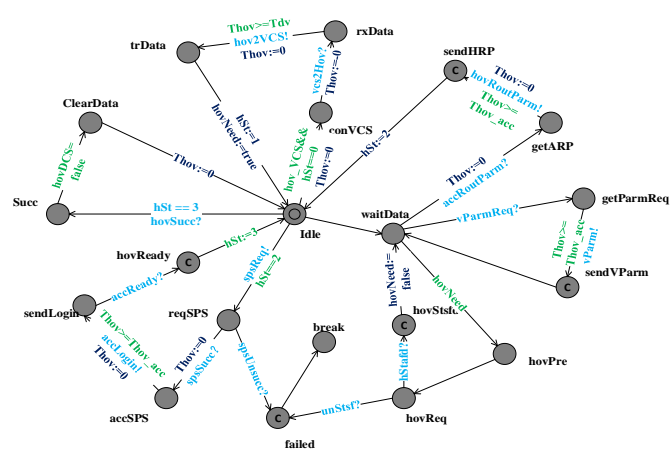

FIGURE IV. TIMED AUTOMATA OF HANDOVER DCS

acceptance DCS, at the end of the handover process, handoverDCS will cut the connection with the train and delete the related data.

As shown in Figure 4, there are four stages in the process. At the beginning $(\mathrm{hSt}=0)$, handover DCS communicate with the VCS periodically via synchronization channels "vcs2Hov?" and "hov2VCS!". In the next stage $(\mathrm{hSt}=1)$ when the train reach the handover zone, it will send handover request to the acceptance DCS by channel "hReq". If the condition satisfy the requirement for handover, it will receive an "hStsd" from acceptance DCS, then 2 DCSs start to exchange vehicle parameter, route parameter via channel "vParmReq", "vParm", "accRoutParm" and "hovRoutParm". While if the condition can't satisfy the requirement, there should be an "unStsf" from acceptance DCS, then it will turn to state Failed. In the third stage $(\mathrm{hSt}=2)$, handover DCS send stop point stepping request to the accDCS via "spsReq", acceptance DCS will reply a "spsSucc" message after stepping successfully or a "spsUnsucc" when there is a failure. Then handover DCS send vehicle login massage via "accLogin". After connected with VCS, acceptance DCS will send a "acc_Ready" message. In the final stage (hSt $=3$ ), after the system handover successfully, the handover DCS receive handover success message via channel "hovSucc" then Boolean variable "acc_VCS" is set to false and the train state is set to "stop".

\section{3) Modeling of acceptance DCS}

The acceptance DCS exchanges massages with handover DCS and establishes communication with VCS, then takes over the protection and control of the train from handover DCS.

As the massage exchange between two DCSs has been described above, there is no need to detail it here. As in Figure 5 , after acceptance DCS receive login massage, it makes the communication between acceptance DCS and VCS accessible by setting the Boolean variable "acc_VCS = true".

\section{B. Verificaton}

This model is designed for single train running through the adjacent decentralized control systems, which can be extended for multi-train. 


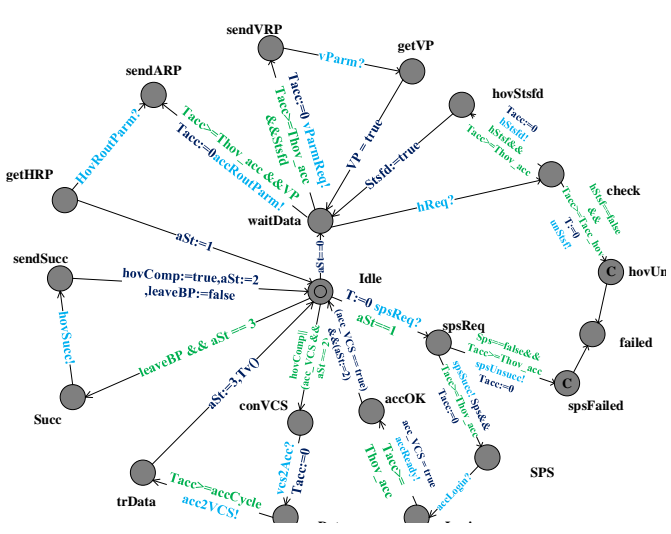

FIGURE V. TIMED AUTOMATA OF ACCEPTANCE DCS

As shown in Figure 6, the interaction between the subsystems can be observed in the simulator of UPPAAL, by which we can make a rough estimate of whether the model meets the system requirement.

Besides, according to the function and performance of the operation control system, some properties must be satisfied to guarantee the system safety and bounded aliveness. Thus for further detail we use BNF (Backus-Naur Form) language to validate those properties, part of which are shown in Figure 7.

- $\mathrm{A}[]$ not deadlock

There is no deadlock in the model

- $\quad \mathrm{E}<>($ accDCS.getHRP)or(accDCS.getVparm)or(hovD CS.getARP)

Acceptance DCS is able to get vehicle parameter from handover DCS and exchange route parameter with it.

- $\mathrm{E}<>(($ VCS.StateVH == 1)and(VCS.StateVA == 1)) VCS is able to connect with both Acceptance DCS and handover DCS.

- $\quad \mathrm{E}<>(($ hovDCS.Succ)and(accDCS.Succ))

The handover process can successfully executed.

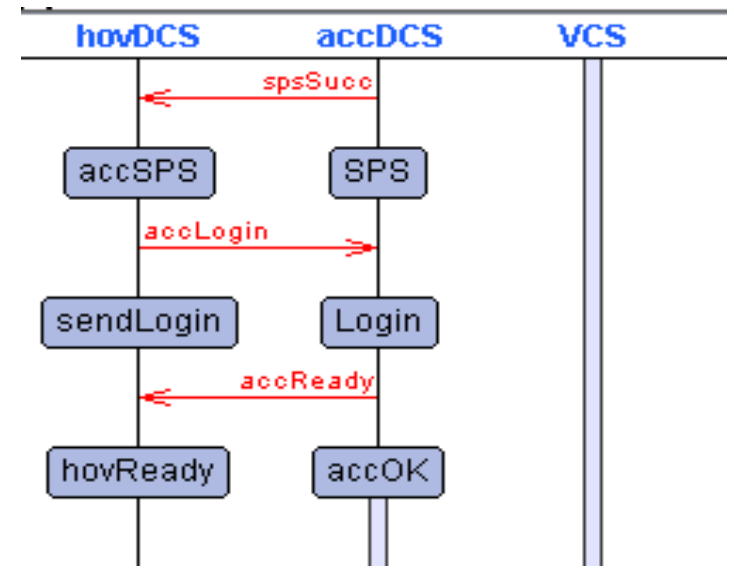

FIGURE VI. RESULTS OF SIMULATION IN UPPAAL

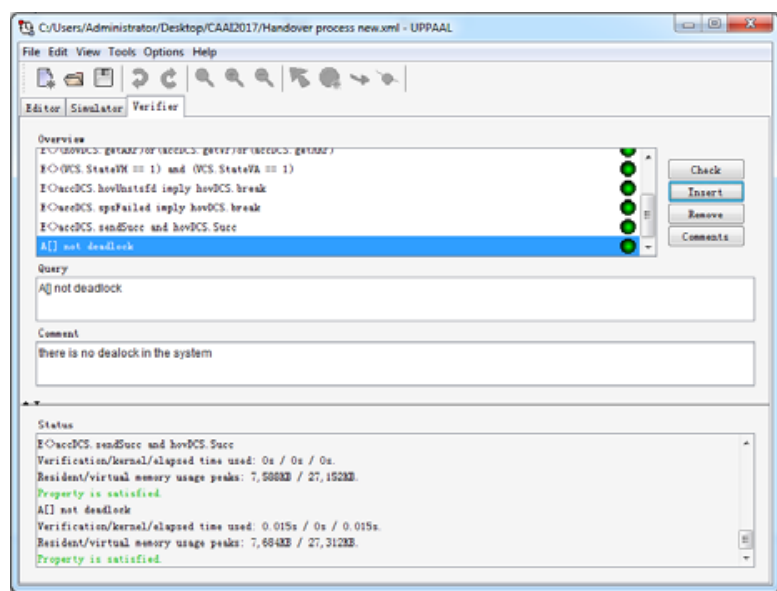

FIGURE VII. VERIFICATION OF PROPERTIES IN UPPAAL

- $\quad$ A [] (accDCS.hovUnstsfd imply hovDCS.break)

A [] (accDCS.spsFailed imply hovDCS.break)

If the Acceptance DCS can't satisfy the handover request or fails to execute stop point stepping, handover DCS should make the train break.

- $\mathrm{A}[]$ (hovDCS.Succ imply $\mathrm{T}<=$ Tmax) units.

The whole handover process should be executed in Tmax

All the properties are validated in the UPPAAL verifier, which implicates the model is able to satisfy the requirements of maglev train operation control system specification.

\section{CONCLUSION}

In this paper, the operation control system of maglev train and its handover process is analyzed. We describe the handover process based on the theory of timed automata by building the automata network of related subsystems using UPPAAL. At last the model is simulated and verified, which successfully validate the safety and bounded aliveness properties of handover process of maglev train operation control system.

\section{REFERENCES}

[1] Wu X M. Maglev Train [J]. Shanghai Science and Technology Press, Shanghai, 2003

[2] Cao Z Q. Modeling of Area Change Module of High Speed Train Based on Colored Petri Net [D]. Fudan University, 2011

[3] Yue An. Formal Modeling and Analysis of RBC Handover Based on Timed UML Sequence Diagram [J]. Railway Standard Design, 2016 (06):132-138

[4] Lu J, Tang T, Jia H. Modeling and Verification of Radio Block Center of CTCS-3 Train Control System for Dedicated Passengers Lines [J]. Journal of the China Railway Society, 2010, 32(6): 35-40

[5] Yang L Q, Zheng Y P. Design of Modeling Method of RBC Handover Based on UML and Colored Petri Nets [J]. Computer Measurement \& Control, 2012, 4: 082

[6] Alur R, Dill D L. A Theory of Timed Automata [J]. Theoretical computer science, 1994, 126(2): 183-235

[7] Bengtsson J, Yi W. Timed Automata: Semantics, Algorithms and Tools $[\mathrm{M}] / /$ Lectures on concurrency and petri nets. Springer Berlin Heidelberg, 2004:87-1 